\title{
Preparation of gelatin scaffold and fibroblast cell culture
}

\author{
Yoshinori Takagi ${ }^{1}$, Susumu Tanaka ${ }^{2}$, Syo Tomita ${ }^{1}$, Shuri Akiyama ${ }^{2}$, Yasuyuki Maki ${ }^{1}$, \\ Takao Yamamoto ${ }^{3}$, Masumi Uehara ${ }^{4}$, Toshiaki Dobashi ${ }^{1, *}$ \\ ${ }^{1}$ Division of Molecular Science, Graduate School of Science and Technology, Gunma University, Kiryu, Gunma 376-8515, Japan \\ ${ }^{2}$ Department of Health and Nutrition, Faculty of Health and Welfare, Takasaki University of Health and Welfare, Takasaki, \\ Gunma 370-0033, Japan \\ ${ }^{3}$ Division of Pure and Applied Science, Graduate School of Science and Technology, Gunma University, Kiryu, Gunma \\ 376-8515, Japan \\ ${ }^{4}$ Graduate School of Radiological Technology, Gunma Prefectural College of Health Sciences, Maebashi, Gunma 371-0052, Japan
}

Received: 7 January 2017 / Accepted: 21 February 2017

(C) Japanese Society of Biorheology 2017

\begin{abstract}
A gelatin gel sheet was UV-irradiated to raise the gel melting temperature $T_{\mathrm{m}}$. Three second of $T_{\mathrm{m}}$ increased proportionally to irradiation time $t_{\mathrm{UV}}$. The sheet of which $T_{\mathrm{m}}$ was larger than the culture temperature $37^{\circ} \mathrm{C}$ was used as a cell culture scaffold. Human fibroblast cells WI-38 adhered to the sheet and dug deeply into it regardless of $t_{\mathrm{UV}}$, while cancer cells WI-38-VA-13 formed spheroids and did not adhere to the sheet individually at high UV dose. The cell selectivity of the scaffold could be used for biological and medical studies.
\end{abstract}

Keywords fibroblast, cell culture, gelatin gel scaffold, UV-irradiation

\section{Introduction}

The melting point of gelatin gels can be raised by cross-linking gelatin molecules in aqueous solutions with UV irradiation [1]. The modified gels can be used for cell culture scaffold, since they are stable at physiological temperature for cell culture [2]. We reported that human fibroblast cells WI-38 can be cultured on a UVirradiated gelatin microcapsule scaffold. Interestingly, in contrast to the normal cells, cancer cells WI-38-VA-13 could not be grown on the microcapsules [3, 4]. This characteristic property could be used for selective cell culture both in vivo and in vitro. A sheet having the same characteristics as the microcapsules would be easier to handle for practical purposes. In this study, we have pre- pared gelatin gel sheets by UV-irradiation, and observed microscopic difference in adhesion of normal and cancer fibroblast cells on the sheet.

\section{Materials and Methods}

\subsection{Preparation of gelatin gel sheet}

Porcine gelatin (APH-250, Nitta Gelatin Inc ) was dissolved in Milli-Q water at $40^{\circ} \mathrm{C}$ at $3 \mathrm{wt} \%$ to make a gelatin solution. $0.5 \mathrm{~mL}$ of the solution was dripped in a PMMA pipe $(24 \mathrm{~mm} \phi \times 10 \mathrm{~mm})$ on a Teflon sheet on ice to make the solution gelled. The physical gel obtained was kept in a refrigerator at $10 \%$ humidity at $5^{\circ} \mathrm{C}$ for 2 days. Both sides of the gel sheet was irradiated with UV ray at $3400 \mu \mathrm{W} / \mathrm{cm}^{2}$ of $254 \mathrm{~nm}$ (CSL-100C, Cosmo Bio Co. Ltd) for $t_{\mathrm{UV}}=0-400 \mathrm{~min}$. The distance between the light source and the sheet was $5 \mathrm{~cm}$. The resultant chemical gel was hydrated in Milli-Q water in a refrigerator for one day and used for measurements.

\subsection{Measurement of melting point}

Each gel sheet prepared with UV irradiation for various period $t_{\mathrm{UV}}$ was put in test tubes, sealed and set in a water bath at $26^{\circ} \mathrm{C}$. The temperature of the bath was then raised at $0.5^{\circ} \mathrm{C} / \mathrm{min}$. The melting point of the gel sheets was determined as the temperature when fluidity was observed by eyes.

*E-mail: dobashi@gunma-u.ac.jp 


\subsection{Cell culture}

Human fibroblast cells (WI-38) and cancer cells (WI38-VA-13) were purchased from JCRB Cell Bank. The cell culture medium was Minimum Essential Medium Eagle containing $10 \mathrm{v} / \mathrm{v} \%$ fetal bovine serum, 50 units $/ \mathrm{ml}$ of penicillin, $50 \mathrm{mg} / \mathrm{ml}$ of streptomycin, an appropriate amount of vitamin and amino acid. Cell culture was performed using the gelatin sheets prepared by UV irradiation for $t_{\mathrm{UV}}$ in the range 30-400 min. The sheets were sterilized in $70 \mathrm{v} / \mathrm{v} \%$ ethanol, and washed three times in phosphate buffer at $\mathrm{pH}=7.4$. For fibroblast cell culture, $1 \mathrm{~mL}$ of the medium was poured in 6 well microtiter plates for suspension cells. Then the sheet was put on each well. $1 \mathrm{~mL}$ of cells whose number concentration was $5 \times 10^{4}$ cells $/ \mathrm{mL}$ was added. The plate was put on an analog shaker for suspension culture. The cells were cultured under $5 \% \mathrm{CO}_{2}$ atmosphere at $37^{\circ} \mathrm{C}$ for 6 days. The shape of the cells and cell aggregates after the culture was observed under an inverted microscope.

\subsection{Electron microscopy}

The sheets prepared with $t_{\mathrm{UV}}=30 \mathrm{~min}$ and $400 \mathrm{~min}$ was fixed by $0.25 \%$ glutaraldehyde in $0.1 \mathrm{M}$ phosphate buffered solution (PBS, pH7.4) for one day. The sheets were washed with $0.1 \mathrm{M}$ PBS and dehydrated with graded ethanol solutions successively. The sheets were further washed with t-butylalcohol and freeze-dried. Finally Au-Pd (Gold-palladium alloy) was sputtered on them by an ion sputter device (E101, Hitachi). The surface structure of the sheets was observed by a scanning electron microscopy (S-2250N, Hitachi). For observation of cancer cells and the sheet near the interface by transmission electron microscope (TEM), the sheets were fixed with $2 \%$ paraformaldehyde and $2 \%$ glutaraldehyde in $0.1 \mathrm{M} \mathrm{PBS}$ at $4{ }^{\circ} \mathrm{C}$ overnight. After this fixation the sheets were postfixed with $2 \%$ osmium tetroxide in 0.1 M PBS at $4{ }^{\circ} \mathrm{C}$ for $2 \mathrm{~h}$, and were dehydrated in graded ethanol solutions $(50 \%, 70 \%, 90 \%$, and $100 \%)$, successively. After dehydration the sheets were infiltrated with propylene oxide (PO) and were put into a 70:30 mixture of PO and resin (Quetol-812: Nisshin EM Co.), then PO was volatilized overnight. The sheets were transferred to a fresh $100 \%$ resin, and were polymerized at $60^{\circ} \mathrm{C}$ for 48 $\mathrm{h}$. The polymerized resin were ultra-thin sectioned at 80 $\mathrm{nm}$ with a diamond knife using an ultramicrotome (Ultracut UCT, Leica) and the sections were mounted on copper grids. They were stained with $2 \%$ uranyl acetate at room temperature for $15 \mathrm{~min}$. The grid was observed by a TEM (JEM-1400Plus, JEOL Ltd.) at an acceleration voltage of $80 \mathrm{kV}$. Digital images were taken with a CCD camera (VELETA, Olympus).

\section{Results and Discussion}

Figure 1 shows the melting temperature as a function of UV irradiation time $t_{\mathrm{UV}}$ for each side. The $3 / 2$ power of melting temperature $T_{\mathrm{m}}$ is linear to $t_{\mathrm{UV}}$, i.e.,

$T_{m} \propto\left(1+\beta t_{U V}\right)^{2 / 3}$

where $\beta$ is a constant which depends only on gelatin weight fraction. This behavior is the same as that of the gelatin gel wall membrane of microcapsules explained by the following theoretical argument [5]. UV irradiation for gelatin induces intermolecular covalent bondings, which results in molecular weight increase. Equation (1) is generally obtained by assuming that (a) the rate of a binding after the UV irradiation is proportional to the number density of the un-reacted functional groups, and (b) the potential energy resulted from a deviation $r$ of a gelatin molecule from its equilibrium position is given by [5]

$U(r)=\frac{1}{2} k\left|r^{2}\right|$

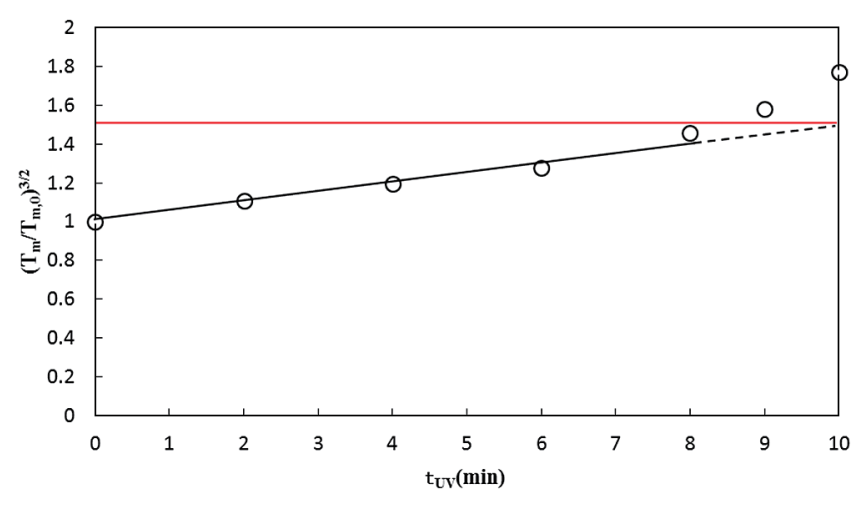

Fig. 1 Melting temperature of gelatin sheet as a function of UV irradiation time. Dotted line denotes $37^{\circ} \mathrm{C}$.

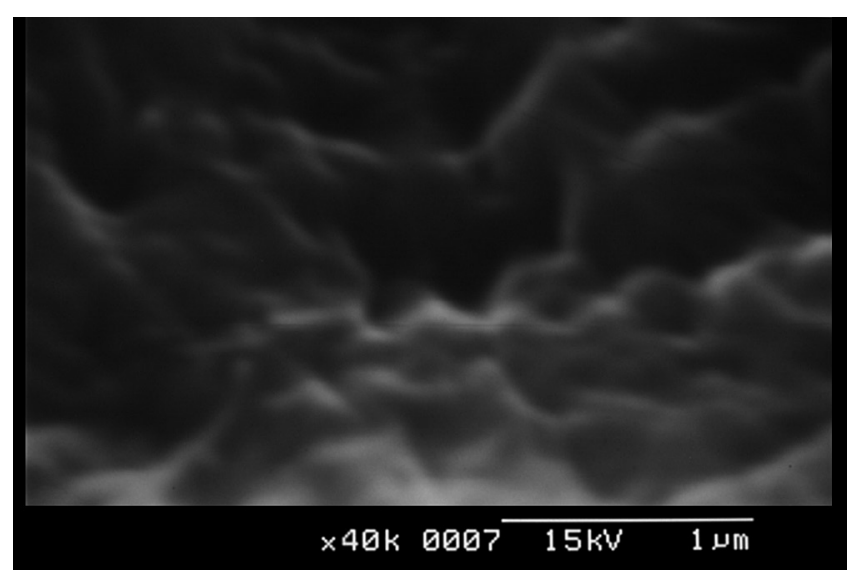

Fig. 2 Scanning electron micrograph of gelatin sheet prepared by UV irradiation for $30 \mathrm{~min}$. 
(A)

(a)

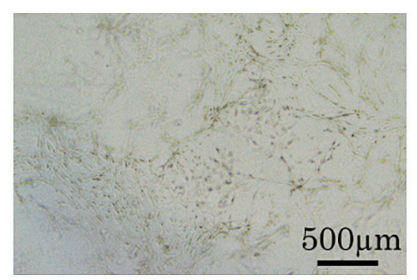

(B)

(a)

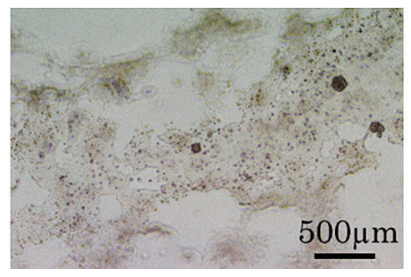

(b)

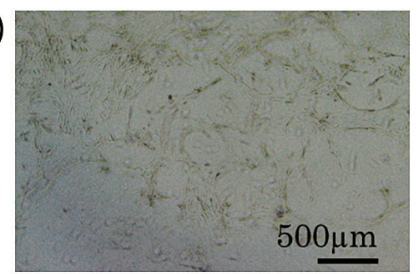

(b)

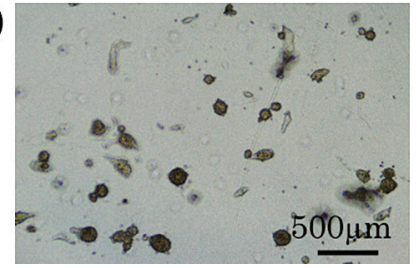

(c)

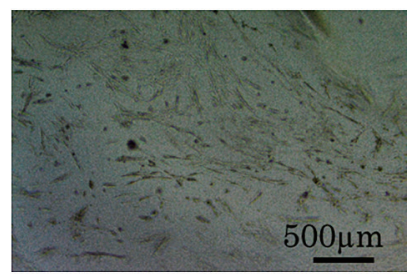

(c)

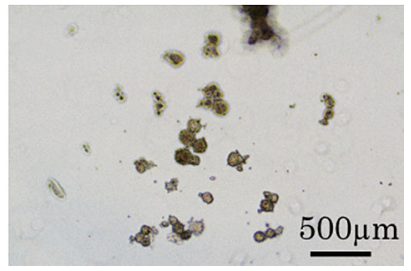

Fig. 3 Optical micrographs of normal cells (WI-38) (A) and cancer cells (WI-38-VA-13) (B) prepared with $t_{\mathrm{UV}}=30 \mathrm{~min}(\mathrm{a}), 40 \mathrm{~min}(\mathrm{~b})$ and 400 $\min (\mathrm{c})$.

(a)

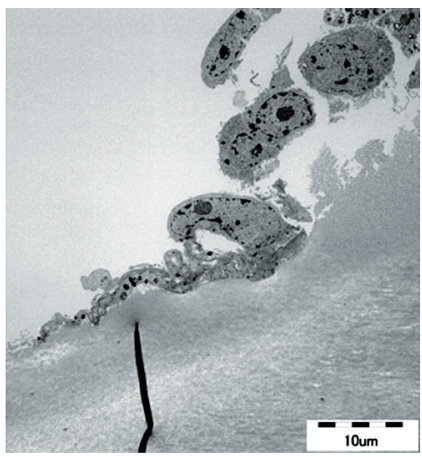

(b)

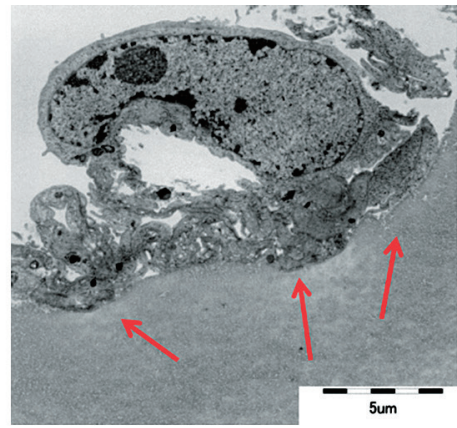

(c)

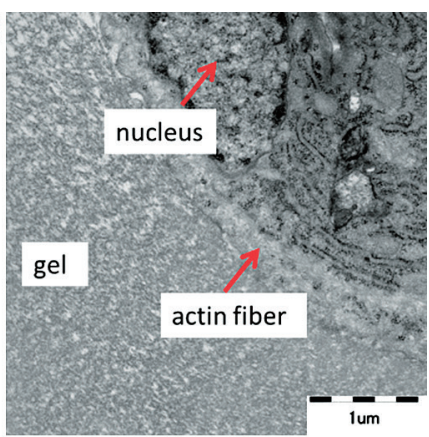

Fig. 4 Transmission electron micrographs near the adhesion surface for WI-38. The magnification is 1760 (a), 4780 (b) and 19000 (c). The cells dig into the gel sheet as shown by red arrows.

(a)

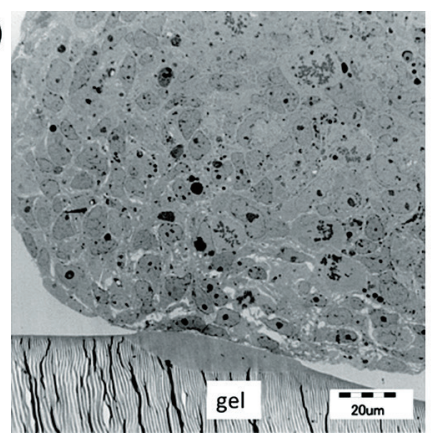

(b)

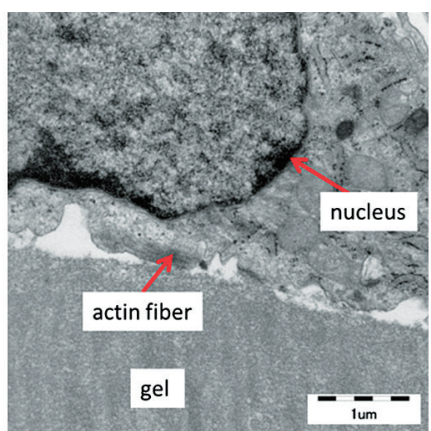

(c)

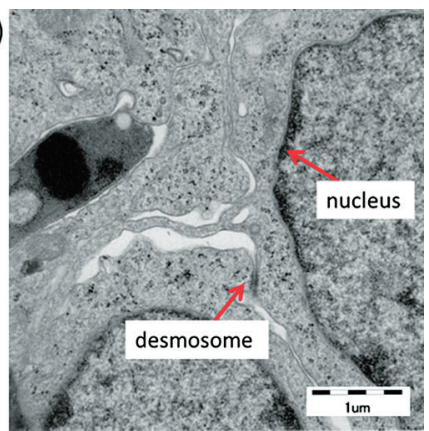

Fig. 5 Transmission electron micrographs near the adhesion surface (a) and (b) and in the cell aggregates (c) for WI-38-VA-13. The magnification is 598 for (a) and 19000 for (b) and (c).

Here, $k$ is the elastic coefficient of the binding, and is proportional to $P^{2 / 3}$ in which $P$ is the degree of polymerization of the gelatin molecule. From (a) for the irradiation time $t_{\mathrm{UV}}$, we have

$$
P=1+\beta t_{U V}
$$

Equation (1) is obtained according to Lindeman's law [6] based on (b). The experimental results show more than 10 min-UV-irradiation is necessary to raise the melting temperature to the culture temperature of $37^{\circ} \mathrm{C}$. Figure 2 shows a scanning electron micrograph of the sheet prepared with UV irradiation at $t_{\mathrm{UV}}=30 \mathrm{~min}$. Slight wavy surface roughness in the order of microns is observed, which could be attributed to an inhomogeneous shrinking and swelling in the process of soaking in ethanol and culture media. No clear difference was observed in the surface structure of the sheets prepared 
with different $t_{\mathrm{UV}}$ (not shown).

Figure 3 shows the microscopic images for normal fibroblast cells WI-38 (A) and cancer cells WI-38-VA-13 (B) on the sheet prepared by UV irradiation for $t_{\mathrm{UV}}$ being $30 \mathrm{~min}$ (a), $40 \mathrm{~min}$ (b) and $400 \mathrm{~min}$ (c). The normal cells are observed to be spreading out homogeneously in plane on the sheet irrespective of $t_{\mathrm{UV}}$. On the other hand, most cancer cells are spreading out in plane on the sheet with $t_{\mathrm{UV}}<30 \mathrm{~min}$, and they form spheroid aggregates and adhere to the gel sheet with a small contact area on the sheet with $t_{\mathrm{UV}}>40 \mathrm{~min}$. More detailed information near the interface between the cell or cell aggregates and the sheet prepared with $t_{\mathrm{UV}}=100 \mathrm{~min}$ is shown as a transmission electron micrographs for normal cells WI-38 in Fig. 4 and cancer cells WI-38-VA-13 in Fig. 5. Figure 4(a) and the enlarged one 4(b) shows that WI-38 cells capture the gel sheet and dig deeply into it. Actin fibers are clearly observed between the cell and the sheet in Figure 4(c). On the other hand, large spheroid aggregates of WI-38-VA-13 cells contact with the gel sheet with a small contact area as shown in Fig. 5(a), but do not dig into the sheet. Figure 5(b) shows actin fibers are formed in the contact cells (Fig. 5(b)). Desmosomes are clearly formed inside the spheroid aggregates, as shown in Fig. 5(c). These experimental results show that cancer cells do not adhere individually to the gelatin sheet modified by UV irradiation with $t_{\mathrm{UV}}>40 \mathrm{~min}$ but tend to form aggregates. WI-38-VA-13 cells synthesize only $1 / 9$ amount of fibronectin compared with WI-38 cells [7]. They secrete a limited amount of fibronectin, but aggregate with each other to form a spheroid to grow. The reason of the difference of observed growth behaviors in WI-38 and WI-38-VA-13 is speculated as follows; WI38 -VA-13 cells adhere to scaffolds having many adhesion sites, while they form a spheroid or adhere with a small contact area on scaffolds having a small number of adhesion sites such as UV-irradiated gelatin scaffold in which many adhesion sites are broken by UV irradiation. While WI-38 cells synthesize and secrete much more amount of fibronectin that enable the cells to adhere to even UV-irradiated gelatin scaffold. It is interesting to examine the cell selectivity characteristics of UV-irradiated gelatin gel sheet for cells of different kinds.

In conclusion, the relationship between UV irradiation time for preparing gelatin gel sheet and gel melting temperature was determined and explained by Lindeman's law. The UV-irradiated gelatin gel scaffold was applied to culture normal fibroblast cells WI-38 and cancer cells WI-38-VA-13. Optical and electron micrographs demonstrated that the cancer cells formed spheroid aggregates on the sheet prepared with large irradiation time but most cancer cells did not adhere to the sheet, in contrast to homogeneous adhesion of normal cells to the scaffold.

Acknowledgements This work was supported by JSPS KAKENHI Grant Number 26560236.

\section{References}

1. For example, Otoni CG, Avena-Bustillos RJA, Chiou BS, BilbaoSainz C, Bechtel PJ, McHugh TH. Ultraviolet-B radiation induced cross-linking improves physical properties of cold- and warmwater fish gelatin gels and films. J Food Sci. 2012; 77: E215-23.

2. Kang HW, Tabata Y, Ikada Y. Fabrication of porous gelatin scaffolds for tissue engineering. Biomaterials. 1999; 20: 1339-44.

3. Dobashi T, Koike M, Kobayashi K, Maki Y, Yamamoto T, Tanaka S. An application of microcapsules having enzyme-degradable gel membrane to cell culture. Progr Colloid Polym Sci. 2009; 136: $149-54$.

4. Koike M, Kobayashi K, Tanaka S, Harano A, Yamamoto T, Dobashi T. Development of cell culture scaffold composed of microcapsule: determination of preparation condition. Trans MRS-J. 2006; 31: 823-5.

5. Yamamoto T, Koike M, Dobashi T. Melting and swelling behaviors of UV-irradiated gelatin gel microcapsules. Langmuir. 2007; 23: 8531-7.

6. Lindeman FA. The calculation of molecular vibration frequencies. Physik Z. 1910; 11: 609-12.

7. Lee BH, Park RW, Sohn KY, Kim IS. Antagonistic regulation of protein kinase $\mathrm{C}$-induced stimulation of fibronectin synthesis by cyclic AMP in human lung fibroblasts. Biochem Mol Biol Int. 1997; 43: 383-90. 